\title{
A Grid-Based Bridging Domain Multiple-Scale Method for Computational Nanotechnology
}

\author{
Shaowen Wang ${ }^{1}$, Shaoping $\mathrm{Xiao}^{2,3}$, and Jun $\mathrm{Ni}^{1,2,4}$ \\ ${ }^{1}$ Academic Technology -- Research Service, Information Technology Services \\ ${ }^{2}$ Department of Mechanical and Industrial Engineering \\ ${ }^{3}$ Center for Computer-Aided Design \\ ${ }^{4}$ Department of Computer Science, \\ The University of Iowa, Iowa city, IA, 52242, USA \\ \{Shaowen-wang, shaoping-xiao, jun-ni\}@uiowa.edu
}

\begin{abstract}
This paper presents an application-level Grid middleware framework to support a bridging domain multi-scale method fornumerical modeling and simulation in nanotechnology. The framework considers a multiple-length-scale model by designing specific domain-decomposition and communication algorithms based on Grid computing. The framework is designed to enable researchers to conductlarge-scale computing in computational nanotechnology through the use of Grid resources for exploring microscopic physical properties of materials without losing details of nanoscale physical phenomena.
\end{abstract}

\section{Introduction}

In the study of nano-materials or nano-devices, a molecular dynamics (MD) model [1] plays an important role. However, many existing MD algorithms have limitations either on length or on time scales due to the lack of enough compute power. Even the most powerful high performance computing (HPC) system is still not powerful enough to perform a complete MD simulation [2]. Therefore, an innovative computational methodology for MD simulations is urgently needed.

Recently-developed concurrent multiscale methods [3] take into consideration of multiple length scales in predicting macroscopic properties. For example, a bridging domain coupling method [4] performs a linkage between the continuum and molecular models. However, if the ratio of mesh size in the continuum domain to the lattice space in the molecular domain is too large, there is a difficult to eliminate a spurious phenomenon in which a nonphysical wave reflection occurs at the interface of different length scales. Therefore, concurrent multiscale methods also require a tremendous computing power. In addition, a small time step (the order of femtosecond) must be selected to meet numerical stability of MD stimulation at the molecular model. Such requirement causes to waste compute time during simulations. A feasible and efficient method is needed to conduct a complete modeling and simulation of large nano systems within long time scales. This requirement stimulates the authors to develop a Grid-based Bridging Domain Multiscale Method (GBDMM). 
Grid computing [5-7] enables users to assemble large-scale, geographicallydistributed, heterogeneous computational resources together for intensive MD computations. This assemblage is dynamically orchestrated using a set of protocols as well as specialized software referred to as Grid middleware. This coordinated sharing of resources takes place within formal or informal consortia of individuals and/or institutions that are often called Virtual Organizations (VO) [8]. In a similar way, Grid application-specific middleware must be developed for MD related multi-scale methods. We intend to develop a micro/macro coupling method and Grid application middleware for multiscale computation in nano-science and technology.

Section 2 reviews the bridging domain coupling method and describes its extensions in the GBDMM. Section 3 addresses domain-decomposition and communication algorithms for the GBDMM. Section 4 presents the framework of Grid nanomiddleware for GBDMM's implementation.

\section{Grid-Enhanced Bridging Domain Multiscale Method (GBDMM)}

\subsection{Bridging Domain Coupling Method}

The bridging domain coupling method contains a continuum domain, $\Omega^{C}$, which is overlapped with a molecular domain, $\Omega^{M}$, through a bridging domain, $\Omega^{\text {int }}$ (Fig. 1). The scaling of the molecular and continuum Hamiltonians is performed in the bridging domain. The Lagrange multiplier method is used to conjunct the molecular domain with the continuum domain via constraint condition(s), $\mathbf{u}^{C}\left(\mathbf{X}_{I}, t\right)-\mathbf{u}_{I}^{M}(t)=0$, where $\mathbf{u}$ is the displacement. The total Hamiltonian of the system can be written as

$$
\begin{aligned}
& \mathrm{H}=\int_{\Omega^{\mathrm{C}}} \beta(\mathbf{X})\left(\mathrm{K}^{\mathrm{C}}+\mathrm{U}^{\mathrm{C}}\right) \mathrm{d} \Omega^{\mathrm{C}}+\sum_{\mathrm{I}}\left(1-\beta\left(\mathbf{X}_{\mathrm{I}}\right)\right)\left(\mathrm{K}_{\mathrm{I}}^{\mathrm{M}}+\mathrm{U}_{\mathrm{I}}^{\mathrm{M}}\right)+ \\
& \sum_{\mathrm{I} \in \Omega^{\text {int }}} \lambda\left(\mathbf{X}_{\mathrm{I}}\right)\left(\mathbf{u}^{\mathrm{C}}\left(\mathbf{X}_{\mathrm{I}}, \mathrm{t}\right)-\mathbf{u}_{\mathrm{I}}^{\mathrm{M}}(\mathrm{t})\right)
\end{aligned}
$$

where $\beta(\mathbf{X})$ equals zero in $\Omega^{M}$ and unity in $\Omega^{C}$ except the bridging domain. Within the bridging domain, $\Omega^{\text {int }}$, it smoothly varies from zero to one. If an irregular bridging domain is considered, a nonlinear function $\beta(\mathbf{X})$ can be introduced. The function can be derived from a signed distance function, or defined by radial basis functions from a set of points. $K$ and $U$ are the kinetic and potential energies. $\lambda(\mathbf{X})$ is a Lagrange multiplier field that can be obtained from the finite element approximation as shown in Fig. 1. Based on the classical Hamiltonian mechanics, the discrete equations of Equation (1) can be expressed as 


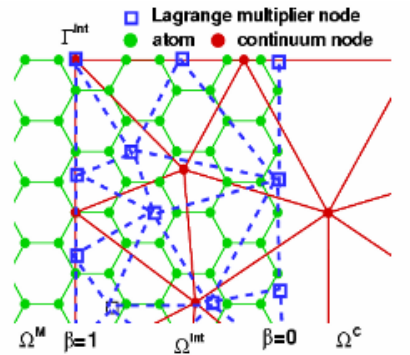

Fig. 1. The bridging domain coupling model for a graphite sheet

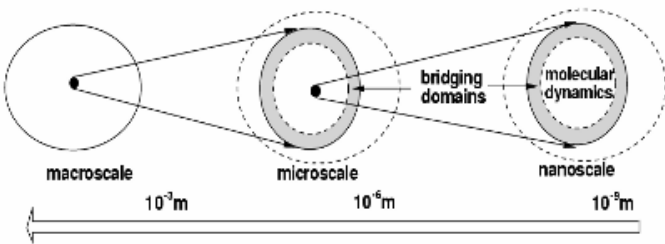

Fig. 2. Length scales covered in the BDMM

$$
\begin{aligned}
& \overline{\mathrm{M}}_{\mathrm{I}} \ddot{\mathrm{u}}_{\mathrm{I}}^{\mathrm{C}}=\mathbf{f}_{\mathrm{I}}^{\mathrm{extC}}-\mathbf{f}_{\mathrm{I}}^{\mathrm{int} \mathrm{C}}-\mathbf{f}_{\mathrm{I}}^{\mathrm{LC}} \quad \text { in } \Omega^{\mathrm{C}}, \\
& \overline{\mathrm{m}}_{\mathrm{I}} \ddot{\mathrm{u}}_{\mathrm{I}}^{\mathrm{M}}=\mathbf{f}_{\mathrm{I}}^{\text {extM }}-\mathbf{f}_{\mathrm{I}}^{\text {int } \mathrm{M}}-\mathbf{f}_{\mathrm{I}}^{\mathrm{LM}} \quad \text { in } \Omega^{\mathrm{M}}
\end{aligned}
$$

where $\bar{M}_{I}$ and $\bar{m}_{I}$ are the modified nodal and atom masses; $\mathbf{f}_{I}^{\text {ext }}$ and $\mathbf{f}_{I}^{\text {int }}$ are the external and internal forces, independently; $\mathbf{f}_{I}^{L}$ is the force due to constraints in the bridging domain. During simulation, velocities are obtained independently in the continuum and molecular domains from Equation (2) without the consideration of constraint forces. Constraint conditions are then used to calculate the Lagrange multipliers. Finally, the constraint forces in Equation (2) are used to correct the nodal/atom velocities in the bridging domain, thus eliminating the wave reflection automatically.

\subsection{Multiscale Considerations in GBDMM}

Based on the bridging domain coupling method, we proposed a Bridging Domain Multiscale Method (BDMM), which bridges from nanoscale to microscale to macroscale (Fig. 2). A bridging domain multiscale model contains a macro-scale domain $\left(\sim 10^{-3} \mathrm{~m}\right)$ as a linear elasticity domain in which linear finite element methods (FEM) can be used. The material properties can be obtained from the Representative Volume Element (RVE) technique with molecular dynamics simulation. The BDMM embeds a micro-scale domain $\left(\sim 10^{-6} \mathrm{~m}\right)$ in the macro-scale domain. It models physical behaviors of materials using either nonlinear FEM [9] or extended FEM [10] (if crack propagation is considered at the microscale). Furthermore, a quasi-continuum technique [11] is implemented with the FE methods at the microscale, so that the constitutive equations can be constructed based on the atomic level energy. A sub-domain in a microscale domain can be treated as a nanoscale (molecular) domain $\left(\sim 10^{-9} \mathrm{~m}\right)$ in which molecular dynamics is employed. The BDMM couples various scales without grading down mesh sizes. Such coupling leads to a straightforward implementation of different time steps via a multiple-time-step algorithm. 


\section{Domain Decomposition and Communication Algorithms}

To perform simulations using GBDMM, an efficient algorithm of domain decomposition must be developed. An entire physical domain with multiple length scales specified can be hierarchically divided into multiple sub-domains. The first generation (FG) sub-domains, such as $\Omega_{01}^{M}$ (see Fig3), are generated based on variation in length scales. These sub-domains are allocated on heterogeneous Grid computing resources. Such first-generational domain decomposition is designed to avoid high network latency, and hence produce a loosely-networked computation on Grids.

Each FG sub-domain can further be divided into a number of second generation (SG) sub-domains being parallel-processed on different computational nodes within a Grid resource. A bridging sub-domain is only shared between two SG sub-domains, which belong to two different length scales, respectively. Although one SG subdomain can be overlapped with two or more SG sub-domains, the bridging subdomains are not overlapped with each other.

There are two types of communications in the GBDMM (see Fig. 4). The interdomain communication among the SG sub-domains takes place within an interconnection within a Grid resource, performing a traditional parallel computation. This computation guarantees the consistency and integrity of SG sub-domains and boundary information for solving the governing equations of atom motions. The procedure for solving equations of atom motion on each Grid cluster is independent. After solving the equations at each time step, communication takes place between two clusters. The bridging coupling techniques are then applied to correct the trial velocities of nodes or atoms in each bridging sub-domain independently.

For example, a simple GBDMM have two sub-domains $\Omega_{A}$ and $\Omega_{B}$ in the molecular domain, allocated to a single Grid resource (often a computing cluster), and $\Omega_{C}$ and $\Omega_{D}$ in the continuum domain, allocated to another Grid resource. The communication between the group of $\Omega_{A}$ and $\Omega_{B}$, and the group of $\Omega_{C}$ and $\Omega_{D}$ takes place on networks in a Grid environment. $\Omega_{A}$ and $\Omega_{B}$ share an atom $E$. Bridging domains $\Omega_{A}^{\text {int }}$ and $\Omega_{B}^{\text {int }}$ perform energy and force calculations. The results from $\Omega_{B}^{\text {int }}$ are used to calculate the body potential functions and inter-atomic forces in $\Omega_{A}$, since $\Omega_{A}$ and $\Omega_{B}^{\text {int }}$ share with same atoms. The size of bridging domains depends on the selected potential functions, especially the cutoff distance for Van der Waals potential functions.

The continuum sub-domains, $\Omega_{C}$ and $\Omega_{D}$ share a boundary node $F$ as shown in Fig. 4. $F^{C}$ and $F^{D}$ represent the same node $F$ in different sub-domains. Unlike inter-domain communications (which do not require bridging domains), the communication between continuum sub-domains, $\Omega_{C}$ and $\Omega_{D}$ exchange internal forces of boundary nodes through the networks among Grid resources. For instance, the internal force of node $F^{C}$, calculated in the sub-domain $\Omega_{C}$ is fetched to the sub-domain $\Omega_{D}$ to form an external force on the node of $F^{D}$, but in an opposite direction. A similar procedure is conducted to pass the internal force of node $F^{D}$ as the negatively external force of node $F^{C}$. Therefore, the motions of node $F$, updated from $\Omega_{C}$ and $\Omega_{D}$ are consistent. 


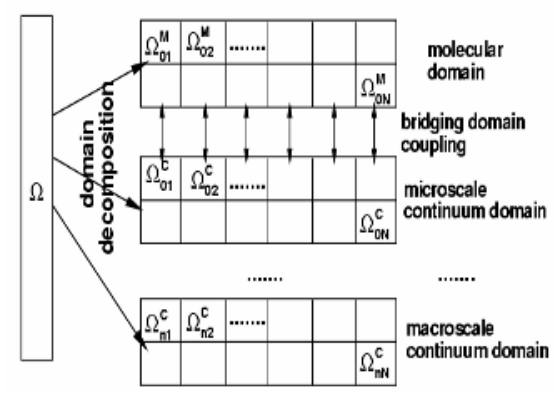

Fig. 3. Domain decomposition for the GBDMM method

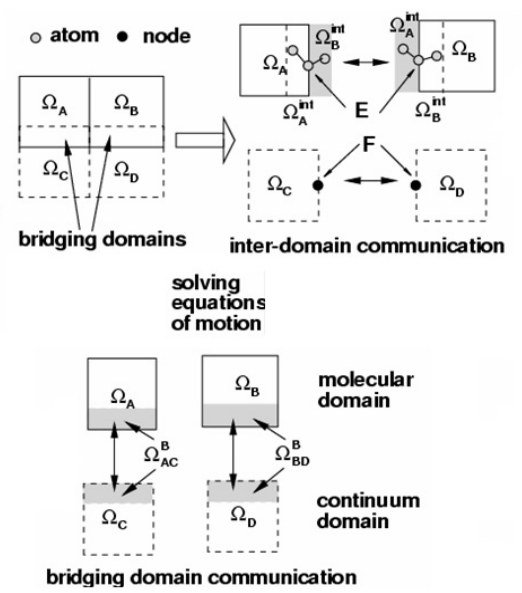

Fig. 4. A demonstration for domain communications

After equations of motion are solved independently on each cluster, bridgingdomain communication takes place independently in each bridging sub-domain, such as $\Omega_{A C}^{B}$ and $\Omega_{B D}^{B}$. For instance, the trial velocities of atoms in $\Omega_{B D}^{B}$ of $\Omega_{B}$ are transferred to $\Omega_{D}$, while the trial velocities of nodes in $\Omega_{B D}^{B}$ of $\Omega_{D}$ are transferred to $\Omega_{B}$. The bridging domain coupling technique is then applied to correct the trial velocities of atoms in $\Omega_{B D}^{B}$ of $\Omega_{B}$ as well as in $\Omega_{B D}^{B}$ of $\Omega_{D}$, respectively on each mater nodes.

\section{Framework of Grid Middleware for GBDMM Implementation}

An application-level Grid middleware framework is designed (in Fig. 5) to enable a GBDMM implementation. In this framework, middleware manages available computing resources, schedules decomposed domains to appropriate Grid resources (i.e. clusters). It contains (1) a task scheduling advisor (TSA) that takes the result of domain decomposition as input to produce scheduling plans and achieve highperformance simulation through load-balancing; and (2) an information broker (IB) that leverages Grid information services to provide the task scheduling advisor with a resource discovery query functions [12].

The framework is centered on the TSA that is used to schedule sub-domains to an appropriate set of Grid resources discovered to achieve optimal performance: tasks are allocated to balance computations across the available set of resources. The subdomains are converted to the tasks that are placed in Grid-resource queues. The TSA is designed to achieve high levels of performance by balancing tasks across available resources. It determines the correspondence between tasks and the available Grid resources. Within the TSA, several static scheduling strategies [13] such as Min-min and Max-min have been implemented. The fine granularity of the domain decomposition in GBDMM is designed to achieve high level parallelism. Static scheduling 
strategies are developed to assign tasks based on computational intensity information for each sub-domain, as well as the variability in the computing capacity of each Grid resource. The workflow of the nano-middleware enhanced GBDMM with Grid computing is shown as Fig. 6.
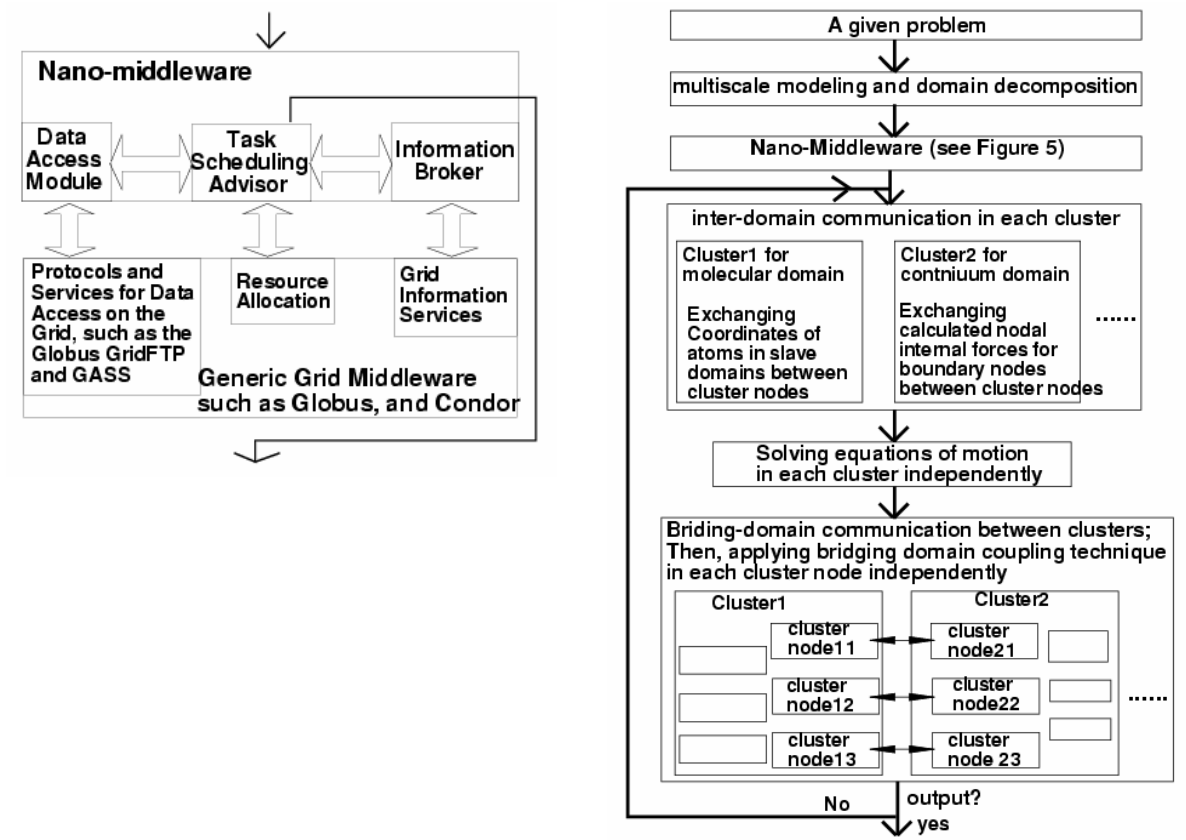

Fig. 5. The Grid nano-middleware architecture. Note: Globus is a software project, the Fig. 6. The work flow of GBDMM purpose of which is to develop protocols and services for computational grids. Condor is a high throughput computing system

TSA is used to schedule sub-domains to an appropriate set of Grid resources discovered to achieve optimal performance: tasks are allocated in a way that balances computations across the available selection of resources. The sub-domains information is converted to the tasks that are placed in Grid-resource queues. The TSA is designed to achieve high levels of performance by balancing tasks across available resources. It determines the correspondence between tasks and the available Grid resources. The TSA deploys both static and dynamic scheduling, planning to operate static and dynamic scheduling on tasks upon the computing recourse available. The TSA dynamically facilitate the swap of tasks between computing resources according to a dynamic performance evaluation. The fine granularity of the domain decomposition in GBDMM is carefully design to achieve high level parallelism. Static scheduling strategies are developed to assign tasks based on computational intensity information for each sub-domain, as well as the variability in the computing capacity of each Grid resource. 
Since Grid resources is very a complex set (resources and network complexities), an IB is needed to perform a self-organized-resource discovery. The IB for the nanomiddleware is designed based on a self-organized grouping (SOG) method that manages the Grid complexity. Therefore, the IB contains three strategies: 1) to control resource heterogeneity during a certain period of time; 2 ) to capture resource dynamics through a publishing mechanism and aggregating mechanism that handles resource dynamically; (3) to enable the assemblage of large number of resources for applications across VO boundaries.

The developed information broker is based on a self-organized resource discovery method. It is well known that currently, the Grid represents an extremely complex distributed computing environment for developing applications because: 1) Grid resources (e.g., CPU, network, storage, and special-purpose instrument) are heterogeneous; 2) Grid resources are dynamic, and they tend to have faults that may not be predictable; 3) Grids are often distributed across security domains with large number of resources involved. Due to this complexity, it has been a great challenge for developing efficient methods for Grid resource discovery that refers to the process of locating satisfactory resources based on user requests. Grid resource discovery must handle the search for desirable subsets of large number of accessible resources, the status and availability of which dynamically change.

The workflow of the nano-middleware enhanced GBDMM with Grid computing is shown as Fig. 6. The workflow demonstrates the integration of computational nanotechnology and computer science in this multi-disciplinary project.

\section{Conclusion and Future Work}

The GBDMM is an extension of the bridging domain coupling method that allows multiple length/time scales to be treated. The GBDMM's domain decomposition and communication are designed to be applicable to distributed Grid resources. The Grid nano-application-middleware includes a task scheduling advisor and an information broker. This middleware enables the GBDM to schedule resources, distribute tasks, and achieve load balancing. Our ongoing work is to implement and apply GBDMM to study mechanical properties of nanostructured materials.

\section{References}

1. Rountree C. L., Kalia R. K., Lidorikis E., Nakano A., Van B. L., Vashishta P: Atomistic aspects of crack propagation in brittle materials: Multimillion atom molecular dynamics simulation. Annual Review of Materials Research 32 (2002) 377-400

2. Abraham F. F., Walkup R., Gao H., Duchaineau M., Rubia T. D., Seager M.: Simulating materials failure by using up to one billion atoms and the world's fastest computer: brittle fracture, Proceedings of the National Academy of Science of the United States of America. 99(9) (2002) 5777-5782

3. Muralidharan K., Deymier P. A., Simmons J. H.: A concurrent multiscale finite difference time domain/molecular dynamics method for bridging an elastic continuum to an atomic system, Modelling and Simulation in Materials Science and Engineering, 11(4) (2003) 487-501 
4. Xiao S. P., Belytschko T.: A bridging domain method for coupling continua with molecular dynamics, Computer Methods in Applied Mechanics and Engineering, 193 (2004) 1645-1669

5. Foster I., Kesselman C.: The Grid: Blue Print for a New Computing Infrastructure. Morgan Kaufmann Publishers, Inc. San Francisco, CA (1999)

6. Foster I.: The Grid: A new infrastructure for 21 st century science, Physics Today (2003) February, 42-47

7. Berman F., Fox G., Hey T.: The Grid, past, present, future, Grid Computing, Making the Global Infrastructure a Reality. edited by R. Berman, G. Fox, and T. Hey John Wiley \& Sons, West Sussex, England (2003)

8. Foster I., Kesselman C., Tuecke S., The anatomy of the grid: enabling scalable virtual organizations, International Journal Supercomputer Applications. 15(3) (2002)

9. Belytschko T., Liu W. K., Moran B.: Nonlinear Finite Elements for Continua and Structures, Wiley, New York (2000)

10. Belytschko T., Parimi C., Moës N., Sukumar N., Usui S.: Structured extended finite element methods for solids defined by implicit surfaces, International Journal for Numerical Methods in Engineering. 56 (2003) 609-635

11. Tadmor E. B., Ortiz M., Phillips R.: Quasicontinuum analysis of defects in solids, Philosophy Magazine A. 73 (1996) 1529-1563

12. Wang, S., Padmanabhan, A., Liu, Y., Briggs, R., Ni, J., He, T., Knosp, B. M., Onel Y.: A multi-agent system framework for end-user level Grid monitoring using geographical information systems (MAGGIS): architecture and implementation. In: Proceedings of Lecture Notes in Computer Science. 3032 (2004) 536-543

13. Braun, T. D., Siegel, H. J., Beck, N., Boloni, L. L., Maheswaran, M., Reuther, A. I., Robertson, J. P., Theys, M. D., Yao, B., Hensgen, D., and Freund, R. F.: A comparison of eleven static heuristics for mapping a class of independent tasks onto heterogeneous distributed computing systems. Journal of Parallel and Distributed Computing. 61(2001) 810-837 